\title{
Compensating springback in the automotive practice using MASHAL
}

\author{
S. Ohnimus, M. Petzoldt, B. Rietman, J. Weiher \\ INPRO Innovationsgesellschaft für fortgeschrittene Produktionssysteme in der Fahrzeugindustrie mbH \\ Hallerstraße 1, D-10587 Berlin, Germany
}

\begin{abstract}
New materials are used in the automotive industry to reduce weight and to improve crash performance. These materials feature a higher ratio of yield stress to elastic modulus leading to increased springback after tool release. The resulting shape deviations and their efficient reduction is of major interest for the automotive industry nowadays. The usual strategies for springback reduction can diminish springback to a certain amount only. In order to reduce the remaining shape deviation a mathematical compensation algorithm is presented. The objective is to obtain the tool geometry such that the part springs back into the right shape after releasing the tools.

In practice the process of compensation involves different tasks beginning with CAD construction of the part, planning the drawing method and tool construction, FE-simulation, deep drawing at try-out stage and measurement of the manufactured part. Thus the compensation can not be treated as an isolated task but as a process with various restrictions and requirements of today's automotive practice. For this reason a software prototype for compensation methods MASHAL - meaning program to maintain accuracy (MASsHALtigkeit) - was developed. The basic idea of compensation with MASHAL is the transfer and application of shape deviations between two different geometries on a third one. The developed algorithm allows for an effective processing of these data, an approximation of springback and shape deviations and for a smooth extrapolation onto the tool geometry.

Following topics are addressed: positioning of parts, global compensation and restriction of compensation to local areas, damping of the compensation function in the blank holder domain, simulation and validation of springback and compensation of CAD-data. The complete compensation procedure is illustrated on an industrial part.
\end{abstract}

\section{INTRODUCTION}

Due to increasing demands for lighter and safer vehicles new materials are adopted in the automotive industry. These materials, e.g. high-strength steels and aluminum, generally tend to show more springback because of their high ratio of yield stress to elastic modulus. During the release phase after sheet forming large deviations between the actual and the desired geometry of the part are observed. So far inaccuracies are compensated manually by reworking the drawing tools in a direction opposite to springback. After forming and release of the compensated tools the part springs back into the desired reference shape.

In this paper a mathematical algorithm is presented which allows for an accurate tool compensation in an automated way. The algorithm is based on the previously mentioned method from practice, known as the displacement adjustment (DA) method. Since this method uses nodal displacement deviations between reference and desired mesh of a preceding finite element simulation compensation is defined in the part region of the tool only. To overcome this problem the smooth displacement adjustment (SDA) method was developed. The shape deviation of the part is calculated and subsequently approximated by an $\mathrm{L}^{2}$ projection of sufficiently smooth functions. Due to the extension to analytical functions the computed shape deviations can easily be transferred to the tool surfaces in order to compensate them. Recent enhancements allow for a compensation on the basis of tactile measurement data as well as reliable control in the addendum and blank holder regions of the tool.

The developed software tool MASHAL was conceived to be applied in an industrial environment. Its functional requirements have been prepared in close cooperation with INPRO's parent companies. A graphical user interface which hides algorithmic details for the user has been implemented within PAMSTAMP 2G. 


\section{ENHANCEMENTS OF THE SDA METHOD}

The SDA method [1,5] is an enhancement of the DA method $[3,4]$. The aim of springback compensation is to define a compensated tool based on a given tool geometry. The tool geometry usually consists of three regions: part, addendum and blank holder region. Given is a discrete displacement field $F$ describing the displacement due to the mainly elastic stress recovery after releasing the tool. This displacement field is defined on a set $P=\left\{p_{i}: p_{i} \in R^{3}\right\}$ of discrete points of the part, for instance the nodes of the part. Thus the discrete displacement field is defined as

$$
F=\left\{\left(p_{i}, u_{i}\right): p_{i} \in P, u_{i} \in R^{3}\right\}
$$

The DA method consists in applying the springback displacement in the opposite direction and multiplying it with a constant factor, the compensation factor $\alpha$ :

$$
c_{i}=p_{i}-\alpha \cdot u_{i}
$$

Obviously, that this geometry defines only the part region of the tool. A modification of the addendum and blank holder region is also needed. This is possible with the SDA method.

\section{The SDA Method}

The SDA method relies on the observation that the displacement field is geometrically a rather smooth function and can therefore be approximated with a low dimensional space of basis functions leading to a sufficiently small approximation error. Instead of using polynomials as described in [1] here the more flexible B-spline functions are used. The approximation space is defined by products of Bspline functions $N_{i}^{x}(x) \cdot N_{j}^{y}(y) \cdot N_{k}^{z}(z)$, where $x$, $y, z$ are defined on a bounding box (parallelepiped) containing the tool. The approximation function is a linear combination of the above basis functions and is determined by minimizing the distance between the discrete displacement field $F$ and the values of the approximation function in the set $P$. As a norm to be minimized the $l^{2}$-norm on the part $P$ is used, leading to a least square minimization problem with component wise solution:

$$
F_{a}(x, y, z):=\sum_{i, j, k} a_{i j k} \cdot N_{i}^{x}(x) \cdot N_{j}^{y}(y) \cdot N_{k}^{z}(z)
$$

As $F_{a}$ is defined on the parallelepiped containing the tool the compensation function

$$
C(x, y, z):=(x, y, z)-\alpha \cdot \mathbf{F}_{a}(x, y, z)
$$

is defined allover the tool in a smooth way. For practical application sometimes a further modification of the compensation function concerning the extrapolation on the blank holder region is required. Examples are shown below.

\section{Advantages of the SDA Method}

As the displacement field is smooth a small amount of input data suffices for approximation. So data from tactile measurements of the sprung back part can be used. Another advantage of the SDA method is that instead of the springback displacement field, which is in fact known only after FEM-simulation, the shape deviation between reference and sprung back part can be used as input. In order to calculate the shape deviation these geometries have to be tessellated. In this case the reference and springback geometry not necessarily have to have a 1-1 node correlation and springback meshes may originate from optical measurement systems. The shape deviation data should not directly be applied to the tool, because this will lead to an unwanted distortion. Instead the SDA method together with a special algorithm implemented in MASHAL recover the springback displacement field to a large extent. When using tactile data a further algorithm takes into account the possibly unequal distribution of measurement points over the part.

\section{APPLICATION OF THE SDA METHOD TO A MOTOR HOOD}

\section{Preparation Of The Compensation}

In order to demonstrate the capabilities of the SDA method the compensation cycle of a motor hood will be presented. The following data have to be read: reference part, springback part and tool geometry. The tool geometry is the tessellated surface used for the FE-simulation of springback and consists usually of die, punch and blank holder. The relative position between reference, springback and tool geometry has 
influence on the compensated geometry. MASHAL provides several methods to determine a coordinate transformation in order to position one geometry with respect to the other. It is also possibly to apply a coordinate transformation defined in the CAD file format VDA.

\section{Compensation With Mesh Data}

Before compensation the user has to choose a compensation factor. An automatic determination of the compensation factor and an iterative approach based on the SDA method is described in [2]. The compensation of the tool geometries: die, punch and blank holder is done within one call of MASHAL. Moreover after choosing the die geometry as "master" the gap between the tools will be maintained. Thus a validating simulation of deep drawing and springback can be carried out afterwards with minimal effort. Figure 1 shows the compensation of a motor hood (courtesy of DaimlerChrysler) with compensation factor 1.0. Additionally the blank holder area was fixed for compensation. One observes that shape deviation for springback and compensation are similar as intended. The approximation error is in general less than $1 \mathrm{~mm}$.

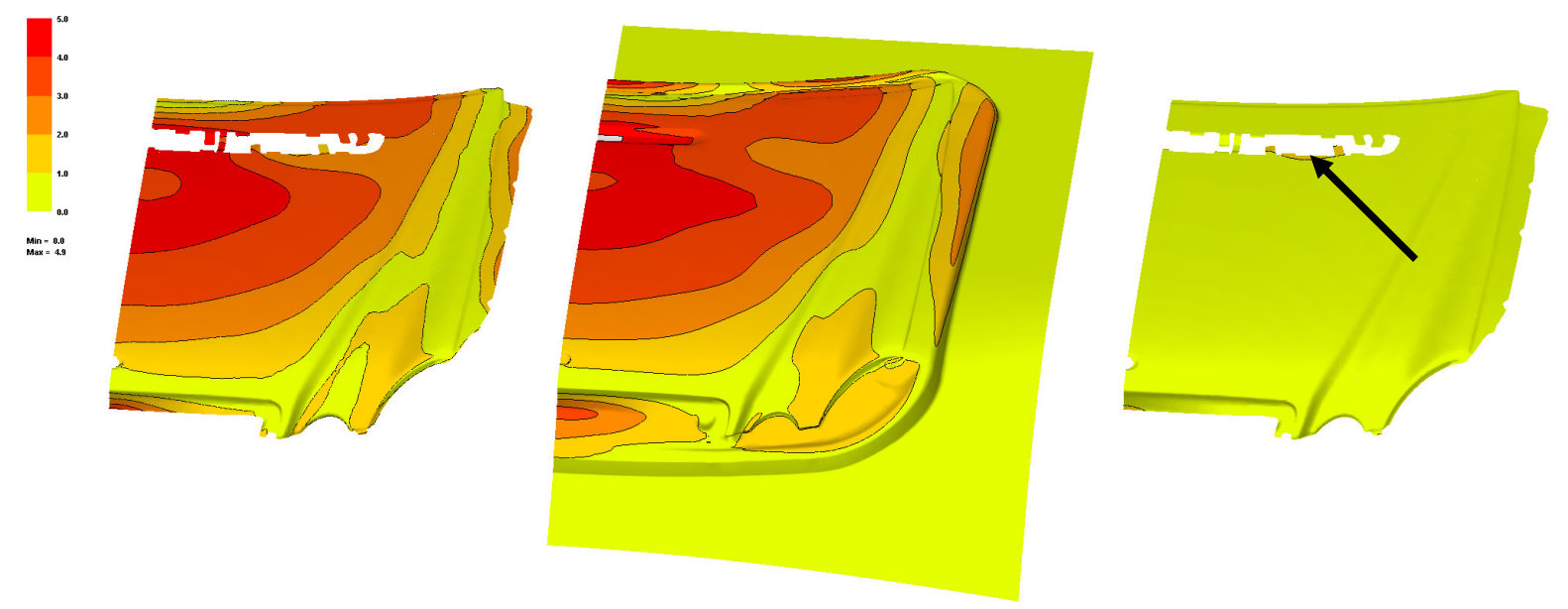

FIGURE 1. a) Shape deviation due to springback up to $5 \mathrm{~mm}$. b) Distance between original and compensated tool is same as shape deviation due to springback. The blank holder area is not changed. c) Approximation error is small allover the part. Maximum reaches $1.6 \mathrm{~mm}$ in flanges (see arrow). All figures with the same scale from 0-5 $\mathrm{mm}$.

There are several special settings for further requirements for the compensation. A symmetry plane can be chosen and the flexibility of the compensation function can be adjusted. The latter results in a different choice of approximating function space. The approximation error is usually lower than $0.5 \mathrm{~mm}$ and can be visualized with MASHAL. Some care has to be taken when interpreting the approximation error (Figure 1c). One has to keep in mind that the SDA method is designed to provide a smooth compensation averaging local shape deviations. In this way locally varying shape deviations and possible errors are smoothed out. These local variations remain as approximation error and have to be interpreted by the user.

\section{Determining The Right Extrapolation}

While the first task of compensation is a good approximation of the displacement field on the part region of the tool, the second task is a good extrapolation of the compensation function onto the addendum and blank holder area not influencing the approximation too much. There is no exact definition what is understood by "good" extrapolation. One possibility of extrapolation is shown in Figure 2. One notices that in this case the compensation introduces local extrema ("buckles"). They can be controlled by filtering out approximating functions with high frequencies. Another way is to extend the displacement field artificially to the blank holder, defining on the latter zero displacement. This reduces compensation on the blank holder region while the 
compensation on the part region of the tool is nearly not influenced. There is also the possibility of canceling the compensation in the blank holder region completely by multiplying with a cut-off function as shown in Figure 3. One observes the desired smooth transition from the part region of the compensated tool with no restriction on the blank holder to the blank holder region of the original tool.

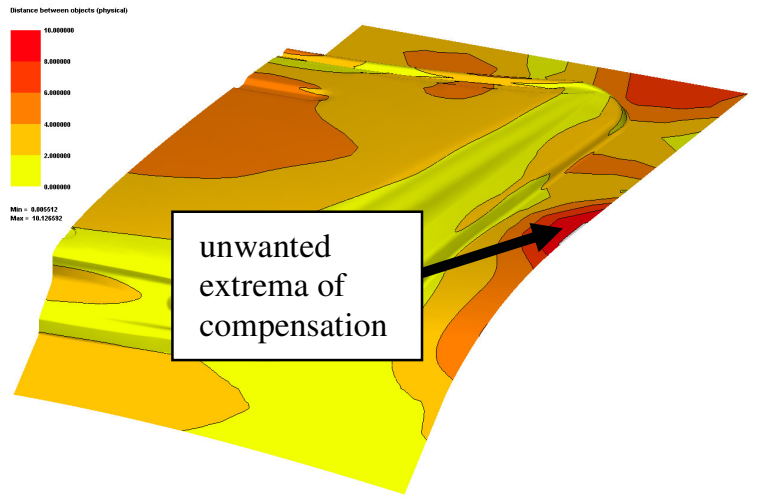

FIGURE 2. Shape deviation between compensated tool and original tool. Possible unwanted compensation in blank holder area. (scale from 0-10 mm)

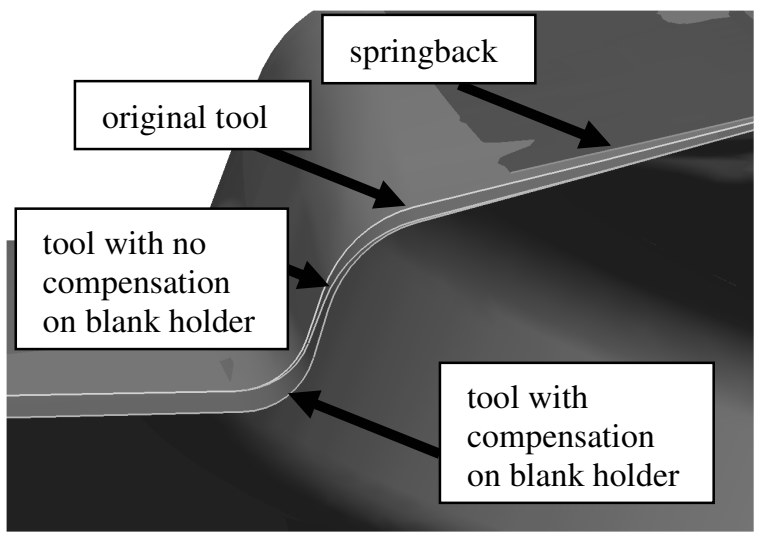

FIGURE 3. Compensation vanishes on the blank holder. A smooth transition of the compensation is obtained.

\section{Compensation With Tactile Data}

The correct prediction of the springback shape via FE-simulation is still a difficult task. But compensation can be assisted on the base of the deep drawn prototype part. In the prototyping phase of tool production prototype parts are measured usually by tactile measurement systems. MASHAL supports different measurement protocols. The shape deviation is determined as the difference between actual and reference measurement points. This displacement field is the basis for calculating the compensation function (Figures 4,5). The compensated tool serves the tool maker for preparing the compensated tool. As for the compensation on the basis of mesh data the SDA method does not exactly match the displacement by shape deviation but approximates it in a smooth way.

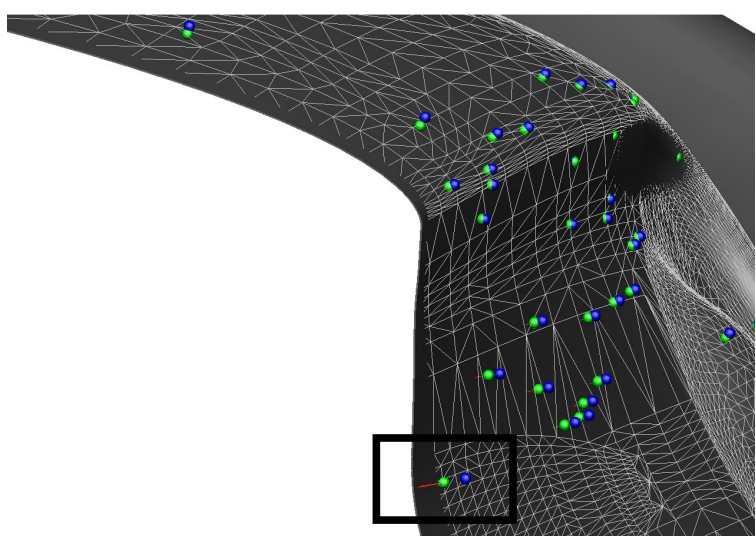

FIGURE 4. Tactile data. Reference points (light gray), actual points (dark gray), original tool (grid), compensated tool (gray).

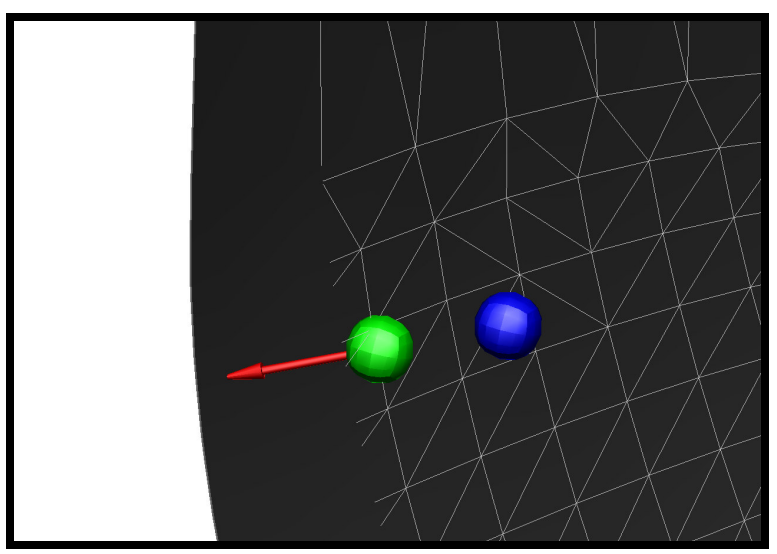

FIGURE 5. Detail of Figure 4. Compensation in opposite direction to springback depicted by the arrow. Reference point (green) is on the original tool (grid) while the actual point is approx. $3 \mathrm{~mm}$ above the original tool.

\section{Compensation of CAD-data}

So far the tool geometries are based on tessellated data. In order to calculate CAD-data of the compensated tool the best way is to apply the displacement of the compensation to the CAD-data of the original tool preserving the original data structure the tool. It is not advised to create CAD-data on the basis of the tessellated data only, since in this case the original structure of the data and a lot of details will be lost. Currently the CAD software systems CATIA and ICEM-Surf are implementing capabilities for applying a displacement field between two topologically equivalent grids to CAD-data. The application of the 
compensation to CAD-data via FE-meshes were tested for a stiff structural part and for an exterior body part. A thorough control of continuity for position, tangents and curvature showed only a small deterioration of the surface quality.

\section{SIMULATION AND COMPENSATION}

Next to the compensation also the prediction of springback is a major issue in sheet forming processes nowadays. The quality and accuracy of a compensation based on finite element calculations on its turn clearly depends on the quality of the simulation. While deep drawing simulations show excellent results for feasibility studies, results are still poor with respect to springback. This must be ascribed to inaccurate stress predictions in the sheet and in thickness direction. The stress-state strongly depends on element kinematics, contact and friction and constitutive behavior.

Commonly used elements in deep drawing simulations are based on shell theory and linear interpolation functions. The reasons for the latter are the simplicity, computation speed and advantages with remeshing, contact, etc. Potentially, however, higher order (shell) elements are more accurate. One of the biggest arguments against higher order elements has always been the unstable contact behavior. A surface contact algorithm instead of the usual nodal contact alleviates this phenomenon. To illustrate this a comparison is presented in Figure 6. In this example a metal sheet is drawn over a tool radius with linear and quadratic shell elements respectively. It can be seen that linear elements with nodal contact are not able to describe the curvature which will negatively affect the development of the internal stresses. In the case of quadratic elements and surface contact the tool curvature is very well described and stress distributions are likely to become smooth.

For the considered materials the standard Hill48 yield criterion may not be sufficient anymore. This has been recognized by the automotive industry and a lot of work is done to develop new descriptions, e.g. based on experimental observations [6,7]. A challenge for the future is the inclusion of micromechanical models to obtain a higher level of accuracy of these phenomenological models.
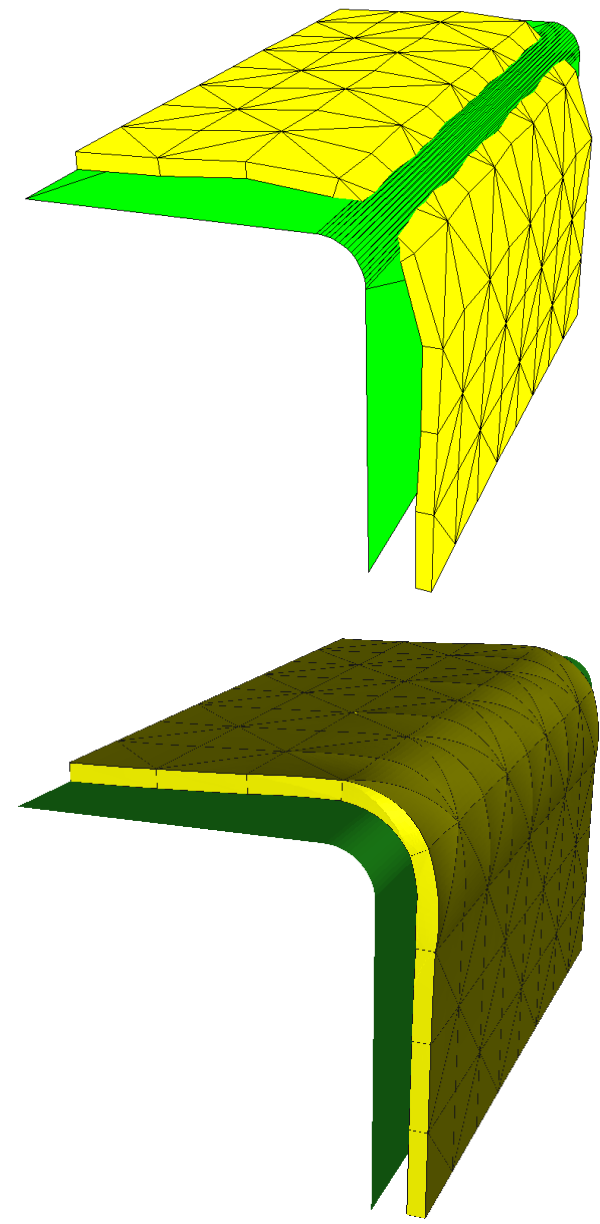

FIGURE 6. Linear thick shells with nodal contact (top) versus quadratic shells with surface contact (bottom).

\section{VALIDATION OF INDUSTRIALIZED FINITE ELEMENT SIMULATION CODES}

The validation of numerical simulations often lacks a thorough approach. In practice the necessary geometry, material and friction parameters and process conditions of the problem are determined on the basis of estimation or measurements. Simulation and experiment are performed independently. Usually a significant difference is observed whose origin remains uncertain. To obtain a better agreement between experiment and simulation a lot of effort is put on additional measurements (e.g. material parameters and geometry) to improve the simulation. This conventional method is therefore basically unidirectional; implicitly it is assumed that the model of the simulation can represent the physics of the 
experiment. This assumption however does not hold considering material fluctuations, tool elasticity, time and space dependent coefficients of friction, element kinematics, etc. Therefore one should ask following questions when large deviations are observed.

Are all of the relevant aspects included in the model?

- A sound verification of mathematical and numerical models must take place.

- A successive comparison of experiment and simulation must take place.

- Possibly the experimental set-up has to be adapted to the capabilities of the simulation model.

Do experiment and simulation base on the same assumptions of the production process?

- The validation must be performed by both the person who carried out the simulation and who carried out the experiment; ideally it's the same person.

In which way the results are evaluated?

- Usually the results of an experiment vary and variations should be measured.

- The result of a simulation should be investigated with respect to changes of input parameters to assess its reliability and significance.

A thorough validation leads to conclusions on:

- Which deviations are due to the simulation model and which are due to the parameters of the model.

- Shortcomings of the model can be identified.

- Reliability and limits of the simulation can be shown.

\section{ACKNOWLEDGEMENTS}

DaimlerChrysler Sindelfingen is greatly acknowledged for providing the data and the knowledge for the hood.
Furthermore the authors wish to thank R. Lingbeek for discussions about practical details and M. Nitsche for implementation of the graphical interface.

\section{REFERENCES}

1. Rietman B., Kose K., Ohnimus S., Petzoldt M., and Weiher J., "Recent Advances in industrially Applied Numerically Aided Springback Compensation" in IDDRG 2004 edited by Kergen R. et al., 2004, pp. 38-44.

2. Lingbeek R.A., Huétink J., Ohnimus S. and Weiher J., "Iterative springback compensation of NUMISHEET benchmark \#1" in NUMISHEET 2005, Conference Proceedings, New York: American Institute of Physics, 2005 (to be published).

3. Gan W., Wagoner R., Mao., Proce S., and Rasouli F., "Practical methods for the design of sheet formed components" in Journal of Material Processing and Technology, 126, 360-367 (2004).

4. Wagoner R., "Fundamental aspects of springback in sheet metal forming" in proceedings ESAFORM 2003 edited by Brucato V., 2003, pp. 7-14

5. Lingbeek R., Huétink H., Ohnimus S., Petzoldt M., and Weiher J. "The development of a finite elements based springback compensation tool for sheet metal products" in Journal of Engineering Materials (accepted for publication).

6. Vegter H., Drent P. and Huétink J., "A planar isotropic yield criterion based on mechanical testing at multi-axial stress states", in proceedings NUMIFORM '95 edited by S.F. Shen and P.R. Dawson, 1995, pp. 345-350.

7. Vegter H. and Van den Boogaard A.H., "A plane stress yield function for anisotropic sheet material by interpolation of biaxial stress states", in International Journal of Plasticity (accepted for publication). 